\title{
Pierre Patrick Haillet (ed.), Procédés de modalisation: l'atténuation
}

\section{Chiara Preite}

\section{(2) OpenEdition}

10 Journals

\section{Édition électronique}

URL : http://journals.openedition.org/studifrancesi/33918

DOI : 10.4000/studifrancesi.33918

ISSN : 2421-5856

Éditeur

Rosenberg \& Sellier

\section{Édition imprimée}

Date de publication : 1 décembre 2005

Pagination : 694-695

ISSN : 0039-2944

\section{Référence électronique}

Chiara Preite, «Pierre Patrick Haillet (ed.), Procédés de modalisation: l'atténuation », Studi Francesi [En ligne], 147 (XLX | III) | 2005, mis en ligne le 30 novembre 2015, consulté le 18 avril 2021. URL : http:// journals.openedition.org/studifrancesi/33918; DOI : https://doi.org/10.4000/studifrancesi.33918

Ce document a été généré automatiquement le 18 avril 2021.

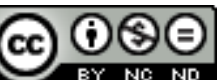

Studi Francesi è distribuita con Licenza Creative Commons Attribuzione - Non commerciale - Non opere derivate 4.0 Internazionale. 


\title{
Pierre Patrick Haillet (ed.), Procédés de modalisation: l'atténuation
}

\author{
Chiara Preite
}

\section{RÉFÉRENCE}

PIERRE PATRICK HAILLET (ed.), Procédés de modalisation: l'atténuation, «Langue française» n.

$142,2004,128 \mathrm{pp}$.

1 Les études réunies dans ce numéro analysent les manifestations de la subjectivité dans le langage sur le mode benvenistien. Les auteurs se sont penchés sur un type particulier de modalisation des énoncés qui exprime la volonté de la part du locuteur de restreindre ou de minimiser la portée de ses propos. Le procédé d'atténuation se manifeste de manière formelle par certains éléments modalisateurs qui traduisent l'attitude du locuteur. D'une part, certains énoncés modalisés (EM) ne diffèrent des énoncés non modalisés (ENM) correspondant qu'à un élément près: syntagme spécifique ou forme verbale. D'autre part, les relations entre l'EM et l'ENM sous-jacent étudiées s'avèrent plus complexes. Dans leur globalité, les études visent à déterminer dans quelles conditions l'emploi d'une forme donnée produit un effet de modalisation.

Pierre Patrick HAILLET, Nature et fonction des représentations discursives: le cas de la "stratégie de la version bémolisée", pp. 7-16.

Cette contribution porte sur l'interprétation d'énoncés du type Il me fallait de la menthe ou J'aurais besoin d'un coup de main, où le choix de telle ou telle forme verbale véhicule une version atténuée, ou «bémolisée», de l'assertion correspondant au présent, qui aurait pu être produite dans les mêmes circonstances: c'est la représentation du besoin en question qui change. Après une présentation du cadre théorique dans lequel son analyse est insérée - l'approche polyphonique où tous les énoncés sont considérés comme des représentations discursives d'au moins un point de vue sur l'objet correspondant - l'auteur décrit la relation qui s'instaure entre la représentation de l'énoncé bémolisé (A) et la représentation de l'énoncé non bémolisé (B) correspondant, 
d'un point de vue formel: B est compatible avec l'environnement discursif de A et A et B sont identiques à la forme verbale près. Le locuteur en tant qu'être du monde tire le bénéfice de l'activité discursive bémolisée du locuteur en tant que tel. Pour conclure, l'auteur esquisse une typologie des énoncés issue de l'approche adoptée.

Danielle LEEMAN, L'emploi de juste comme adverbe d'énonciation, pp. 17-30. Dans cette étude l'auteur remarque que juste, employé adverbialement, peut être envisagé comme un «adverbe d'énonciation», utilisé par le locuteur afin de minimiser la portée de l'énoncé aux yeux de l'interlocuteur ainsi que de devancer la conclusion négative que l'on pourrait en tirer. Bien que non attesté par les vocabulaires consultés et que la syntaxe ne corrobore pas l'intuition sémantique, LEEMAN avance l'hypothèse d'un emploi atténuatif de l'adverbe juste, qui consiste à présenter le contenu de l'énoncé comme n'ayant pas l'importance que l'interlocuteur voudrait lui attribuer. Cette minimisation ne pose pas l'énoncé sous un jour défavorable, mais elle amène l'interlocuteur à une conclusion positive, moyennant une «inversion argumentative». À soutien de sa thèse, l'auteur propose de remplacer une classification syntaxique basée sur des éléments formels avec une approche qui essaye d'interpréter les liens qui s'instaurent entre propriétés syntaxiques et sémantiques, de manière à expliquer les comportements formels inattendus par rapport aux classements existants.

André BoRILLO, Les «Adverbes d'opinion forte» selon moi, à mes yeux, à mon avis,...: point de vue subjectif et effet d'atténuation, pp. 31-40. À l'intérieur de la catégorie des "Adverbes de modalité épistémique» - qui font à leur tour partie du groupe des «Adverbes disjonctifs d'attitude» - l'auteur distingue et regroupe une douzaine de structures adverbiales exprimant la conviction personnelle acquise à travers les croyances, l'appréciation, le jugement, etc., qu'il appelle "Adverbes d'opinion forte», capables de remplir une fonction pragmatique d'atténuation. Par ces expressions adverbiales - qui apparaissent le plus souvent dans des constructions parenthétiques et à la présence d'un élément déictique de $1^{\text {re }}$ personne - le locuteur affaiblit la portée du propos qu'il énonce, en manifestant de la prudence par rapport à la vérité qu'il avance et aux jugements évaluatifs qu'il introduit dans son discours. De cette manière, le locuteur revendique la part de subjectivité de son propos et en relativise la portée, ce qui lui permet de négocier, de se donner une position de repli, éventuellement de se rétracter, sans perdre la face, dans le cas où son propos ne ferait pas l'accord de son interlocuteur

Danielle COLTIER et Patrick D endAle, La modalisation du discours de soi: éléments de description sémantique des expressions pour moi, selon moi et à mon avis, pp. 41-57. Les auteurs étudient et posent en comparaison trois expressions de modalisation discursive de la part du locuteur qui appartiennent à la catégorie des marqueurs de «modalisation en discours second»: selon $N$, pour $N$ et à son avis, qualifiés de «médiatifs» ou d'«évidentiels». En général, ces trois expressions, utilisées de manière exophrastique accompagnées d'un pronom personnel ou d'un adjectif possessif de la première personne - montrent une toute autre fonction que de rapporter un discours autre. Elles expriment une "vue de l'esprit», bien qu'en présentant des sémantismes différents manifestés par des différentes conditions d'usage. Ainsi, pour moi introduit une vue de l'esprit personnelle et parfois déliée de la réalité; en revanche, à mon avis et selon moi se relient à la réalité, mais si à mon avis exprime un point de vue improvisé en réaction à une situation, selon moi manifeste une prise de position soupesée et pondérée, donc plus péremptoire. Il s'ensuit que les images du moi que donnent les trois expressions sont sensiblement différentes. 
7 Lotfi ABOUDA, Deux types d'imparfait atténuatif, pp. 58-74. À partir d'une collecte de données dans des bases de données informatisées, l'auteur remarque que n'importe quel verbe à l'imparfait (IMP) ne permet pas la production d'un effet d'atténuation et il essaye de vérifier quels sont les verbes qui permettent cette modalisation ainsi que leurs propriétés syntaxiques. Un examen détaillé des conditions syntaxiques et discursives des énoncés atténuatif montre, entre autres, que les IMP employés de manière atténuative gardent une certaine "épaisseur temporelle», puisqu'ils semblent renvoyer à un moment du passé où «les raisons de l'intrusion du locuteur étaient au stade de simples intentions», et qu'ils s'emploient de préférence, mais non exclusivement, la $1^{\text {re }}$ personne. Cette analyse permet à l'auteur de remarquer que les énoncés qui contiennent un IMP en substitution d'un présent - ce qui relève d'une modalité discursive d'atténuation - peuvent être repartis en deux sous-catégories selon les propriétés syntaxiques auxquelles ils ont recours. Ainsi, il établit l'existence d'un IMP «déperformatif» qui vise la neutralisation d'un acte de discours donné sur le plan énonciatif et qui est dépourvu de toute chronologie, et d'un IMP «justificatif» qui fournit une justification à l'intrusion du locuteur dans la sphère de l'interlocuteur, par le biais de ses propriétés temporelles et aspectuelles habituelles.

8 Jean-Claude ANSCOMBRE, L'imparfait d'atténuation: quand parler à l'imparfait, c'est faire, pp. 75-99. Cet article vise à fournir une analyse temporelle et aspectuelle détaillée des valeurs modales véhiculées par l'imparfait d'atténuation (IA). ANSCOMBRE opère une classification heuristique qui comprend quatre emplois principaux de l'imparfait: (A) un imparfait d'atténuation proprement dit (questions sous la forme assertive) qui est considéré comme la manifestation standard de l'IA; (B) un imparfait commercial (questions sous la forme interrogative); (C) un imparfait ludique (affirmations destinées à se réaliser dans le futur); (D) un imparfait hypocoristique (commentaire d'une situation actuelle sous la forme d'une affirmation ou d'une question de confirmation). Cette typologie permet d'étudier les caractéristiques linguistiques de l'IA pour en arriver à montrer que seulement $\mathrm{A}$ et $\mathrm{B}$ possèdent certaines valeurs distinctives communes et que les séries $\mathrm{C}$ et $\mathrm{D}$, au contraire, ne peuvent pas être réduites à la série A. Ensuite, l'auteur montre que l'imparfait de type A et B, tout en étant employé pour poser des questions "au sens flou», est tout aussi temporel que ses emplois standard, dont les valeurs modales découlent par le biais de certains principes pragmatiques, à l'intérieur du cadre théorique des marqueurs des actes de discours.

9 Anne-Marie LILTI, Négation d'un terme marqué et procédés de modalisation, pp. 100-111. L'auteur réfléchit sur la valeur d'atténuation attribuée à certains emplois de la négation dans des énoncés du type Ce n'est pas idiot ou Je ne te hais point interprétés en relation avec les «assertions positives équivalentes» - et non correspondantes comme le seraient C'est idiot et Je te hais - C'est intelligent et Je t'aime, où l'adjectif ou le verbe «non marqué» remplace celui - «marqué» - de l'énoncé négatif. Ces énoncés négatifs, tout en provoquant une «impression d'atténuation» perçue par les sujets parlants, ne fonctionnent pas comme des litotes, car ils possèdent la même force et la même orientation argumentative que leurs équivalents. De plus, les autres emplois de la négation d'un terme marqué expriment de manière systématique une stratégie de modalisation, en ce qu'ils montrent l'attitude du locuteur par rapport au point de vue qui correspond à l'énoncé affirmatif présentant le terme non marqué. Cela apparaît clairement à travers le lien qui s'instaure avec la démarche concessive, où la négation du terme marqué réduit la distance entre les deux arguments et accentue leur 
compatibilité. Enfin, l'auteur montre que la stratégie de l'énoncé négatif doit être placée dans une perspective polyphonique car elle met à distance l'assertion positive équivalente dans des interactions du type «question - réponse».

10 Caroline foullioux et Didier TEJEDOR DE FELIPE, À propos du mode et de l'atténuation, pp. 112-126. Les auteurs considèrent que le phénomène de l'atténuation relève d'une stratégie de modalisation qui s'insère dans le cadre théorique de la polyphonie ainsi que de l'«argumentation dans la langue» d'Anscombre et Ducrot. Dans le sillage de ces théories, FOULLIOUX ET TEJEDOR DE FELIPE mènent une recherche circonscrite aux énoncés appartenant aux modes du savoir en espagnol. D'abord, ils reformulent le concept de «mode verbal» en fonction de paramètres (savoir/ne pas savoir, exister/ne pas exister et devoir épistémique/pouvoir épistémique/paraitre) liés au point de vue du locuteur. Ensuite, ils abordent une description de la notion d'atténuation qui implique la présence dans les énoncés de certains marqueurs aptes à produire cet effet, qui consiste à remplacer une représentation discursive par une autre représentation perçue comme moins agressive et présentant un procès comme désactualisé par rapport au moment de l'énonciation. Il s'ensuit que dans tout énoncé atténué s'inscrivent des points de vue par rapport auxquels les locuteurs prennent position. Ces hypothèses sont testées sur des exemples relevant des trois modes dérivés du savoir (réel, réalisable et irréel) qui montrent l'exploitation de marqueurs distincts. 